\title{
Host cell response and distinct gene expression profiles at different stages of Chlamydia trachomatis infection reveals stage-specific biomarkers of infection
}

Emmanuel Enoch Dzakah ${ }^{1,2}$, Liping Huang ${ }^{3}$, Yaohua Xue ${ }^{1}$, Shuai Wei ${ }^{4}$, Xiaolin Wang ${ }^{4}$, Hongliang Chen ${ }^{5}$, Jingwei Shui ${ }^{3}$, Foster Kyei ${ }^{2}$, Farooq Rashid ${ }^{1}$, Heping Zheng ${ }^{1}$, Bing Yang ${ }^{1}$ and Shixing Tang ${ }^{1,3^{*}}$

\begin{abstract}
Background: Chlamydia trachomatis is the most common sexually transmitted infection and the bacterial agent of trachoma globally. C. trachomatis undergoes a biphasic developmental cycle involving an infectious elementary body and a replicative reticulate body. Little is currently known about the gene expression dynamics of host cell mRNAs, IncRNAs, and miRNAs at different stages of $C$. trachomatis development.

Results: Here, we performed RNA-seq and miR-seq on HeLa cells infected with C. trachomatis serovar $\mathrm{E}$ at $20 \mathrm{~h}$ post-infection (hpi) and 44 hpi with or without IFN- $y$ treatment. Our study identified and validated differentially expressed host cell mRNAs, IncRNAs, and miRNAs during infection. Host cells at 20 hpi showed the most differential upregulation of both coding and non-coding genes while at $44 \mathrm{hpi}$ in the presence of IFN- $\gamma$ resulted in a dramatic downregulation of a large proportion of host genes. Using RT-qPCR, we validated the top 5 upregulated mRNAs and miRNAs, which are specific for different stages of $C$. trachomatis development. One of the commonly expressed miRNAs at all three stages of $C$. trachomatis development, miR-193b-5p, showed significant expression in clinical serum samples of $C$. trachomatis-infected patients as compared to sera from healthy controls and HIV-1-infected patients. Furthermore, we observed significant upregulation of antigen processing and presentation, and $\mathrm{T}$ helper cell differentiation pathways at 20 hpi whereas T cell receptor, mTOR, and Rap1 pathways were modulated at 44 hpi. Treatment with IFN- $\gamma$ at 44 hpi showed the upregulation of cytokine-cytokine receptor interaction, FoxO signaling, and Ras signaling pathways.
\end{abstract}

Conclusions: Our study documented transcriptional manipulation of the host cell genomes and the upregulation of stage-specific signaling pathways necessary for the survival of the pathogen and could serve as potential biomarkers in the diagnosis and management of the disease.

Keywords: Chlamydia trachomatis, Differentially expressed genes, Biomarkers, Signaling pathways, Replication

\footnotetext{
* Correspondence: tamgshixing@smu.edu.cn

${ }^{1}$ Dermatology Hospital of Southern Medical University, Guangzhou, China

${ }^{3}$ Guangdong Provincial Key Laboratory of Tropical Disease Research, School

of Public Health, Southern Medical University, Guangzhou, China

Full list of author information is available at the end of the article
}

(c) The Author(s). 2021 Open Access This article is licensed under a Creative Commons Attribution 4.0 International License, which permits use, sharing, adaptation, distribution and reproduction in any medium or format, as long as you give appropriate credit to the original author(s) and the source, provide a link to the Creative Commons licence, and indicate if changes were made. The images or other third party material in this article are included in the article's Creative Commons. licence, unless indicated otherwise in a credit line to the material. If material is not included in the article's Creative Commons licence and your intended use is not permitted by statutory regulation or exceeds the permitted use, you will need to obtain permission directly from the copyright holder. To view a copy of this licence, visit http://creativecommons.org/licenses/by/4.0/. The Creative Commons Public Domain Dedication waiver (http://creativecommons.org/publicdomain/zero/1.0/) applies to the data made available in this article, unless otherwise stated in a credit line to the data. 


\section{Background}

Chlamydia trachomatis (C. trachomatis) is an obligate intracellular bacterial agent that is considered as one of the most widespread sexually transmitted infections (STIs). C. trachomatis is usually transmitted through risk behaviors similar to human immunodeficiency virus type one (HIV-1) infection in humans [1-4]. Annually, more than 127 million cases of $C$. trachomatis infection occur worldwide [1]. C. trachomatis generally undergoes a biphasic developmental cycle involving an infectious elementary body (EB) and a replicative form called the reticulate body $(\mathrm{RB})[5,6]$. When the EBs get into contact with the host cell, they are internalized to form membrane-bound bodies (inclusion bodies). The EBs then differentiate into RBs that undergo binary fission forming large vacuoles in the host cell. Under unfavorable conditions, such as in the presence of IFN- $\gamma$, the pathogen differentiates into a quiescent noninfectious aberrant structure termed the "persistent" form or aberrant reticulate bodies (ARB) [7-9].

The developmental cycle of $C$. trachomatis is largely dependent on the host cell since the pathogen derives its nutrients from the host cell leading to changes in protein synthesis and host cell signaling pathways $[6,10]$. Thus, the effective manipulation of the host cell's metabolic and immune systems is crucial to the intracellular survival of $C$. trachomatis $[11,12]$. The host cell genome responds to these changes by altering the expression of essential genes and cytokines to maintain proper function and survival of cells, as well as playing important roles in the clearance of the pathogen [13-15]. Inhibition of protein synthesis leads to the reprogramming of the host cells during C. trachomatis infection $[15,16]$. Dramatic changes in the host cell genomes may result in a different outcome of antibiotic treatment of $C$. trachomatis and the existence of the persistent forms of the bacteria. Additionally, it was suggested that both chlamydial and host genes may synergistically function during host cell invasion [6]. Previous studies have mainly focused on Chlamydial transcriptomes over the developmental cycle in different chlamydial species [17-19]. Epithelial cell transcriptome in response to plasmidbearing/plasmid-less $C$. trachomatis was previously characterized by microarray [20]. A recent report also indicated that C. trachomatis-infected host cells exhibit changes in protein synthesis and other host cell signaling pathways [21, 22].

There is currently a limited understanding of the changes in the expression levels of both protein-coding and non-coding genes in host cells at the different stages of $C$. trachomatis infection. The application of nextgeneration sequencing (NGS) techniques may provide a more accurate host cell genome-wide characterization of both coding and non-coding genes at various stages of infection. Here, we infected Hela cells with C. trachomatis and collected the inclusion body-containing host cells at 20 and $44 \mathrm{~h}$ post-infection (hpi), and also in the presence of interferon-gamma (IFN- $\gamma$ ) at $44 \mathrm{hpi}$. Using RNA-seq and miR-seq, the dysregulated host cell mRNAs, lncRNAs, and miRNAs, as well as the activated signaling pathways were documented in C. trachomatisinfected host cells at $20 \mathrm{hpi}$ and $44 \mathrm{hpi}$ with or without IFN- $\gamma$ treatment. Analysis of serum miRNAs in healthy, C. trachomatis-infected, and HIV-1-infected patients showed a significant expression of miR-193b-5p only in C. trachomatis-infected patients. These findings highlight the gene expression dynamics and the dysregulation of $C$. trachomatis stage-specific signaling pathways in host cells during $C$. trachomatis infection and provide insights into potential biomarkers for improved clinical diagnosis and management of $C$. trachomatis infection.

\section{Results \\ Host gene expression patterns during C. trachomatis infection}

To understand how C. trachomatis-infected host cells respond to infection at the various stages of $C$. trachomatis development, we infected HeLa cells with C. trachomatis serovar E. Cells were harvested at $20 \mathrm{hpi}, 44$ hpi without IFN- $\gamma$ treatment or 44 hpi with IFN- $\gamma$ treatment, mimicking the RB, EB, and ARB stages of C. trachomatis development, respectively (Fig. 1a). Total RNA extraction was followed by RNA-seq and miR-seq. Gene expression dynamics revealed significant dysregulation of host cell mRNAs, lncRNAs, and miRNAs at $20 \mathrm{hpi}$, $44 \mathrm{hpi}$, and at $44 \mathrm{hpi}$ with IFN- $\gamma$ treatment as compared to the corresponding uninfected controls. A total of 3260 differentially expressed genes (DEGs) comprising of 2374 (72.8\%) upregulated and 886 (27.2\%) downregulated genes were identified in C. trachomatis-infected cells at 20 hpi (Fig. 1b, e-g; Supplementary Fig. 1a). A total of 2663 DEGs including 1795 (67.4\%) upregulated and 868 (32.6\%) downregulated mRNAs were identified from host cells infected at 44 hpi compared with uninfected controls (Fig. 1c, e-g; Supplementary Fig. 1b). At 44 hpi with IFN- $\gamma$ treatment, we observed 841 DEGs with 355 (42.2\%) upregulated and 486 (57.8\%) downregulated mRNAs (Fig. 1d-e; Supplementary Fig. 1c). Interestingly, 298 upregulated mRNAs were shared between host cells after $20 \mathrm{hpi}$ and $44 \mathrm{hpi}$ as compared to 80 mRNAs at $44 \mathrm{hpi}$ with or without IFN- $\gamma$ treatment, and only 33 mRNAs shared by the host cells at $20 \mathrm{hpi}$ and IFN- $\gamma$ treatment at $44 \mathrm{hpi}$. A total of 2014, 1388, and 213 mRNAs were specifically upregulated at $20 \mathrm{hpi}, 44$ hpi without IFN- $\gamma$, and $44 \mathrm{hpi}$ with IFN- $\gamma$ treatment, respectively (Fig. 1f). Of note, only 29 upregulated and 63 downregulated mRNAs were observed in C. trachomatis-infected host cells in all three treatments. The most 


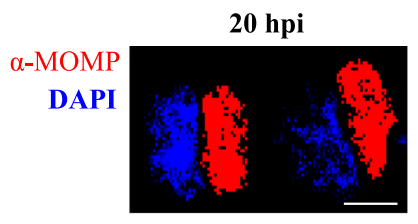

b
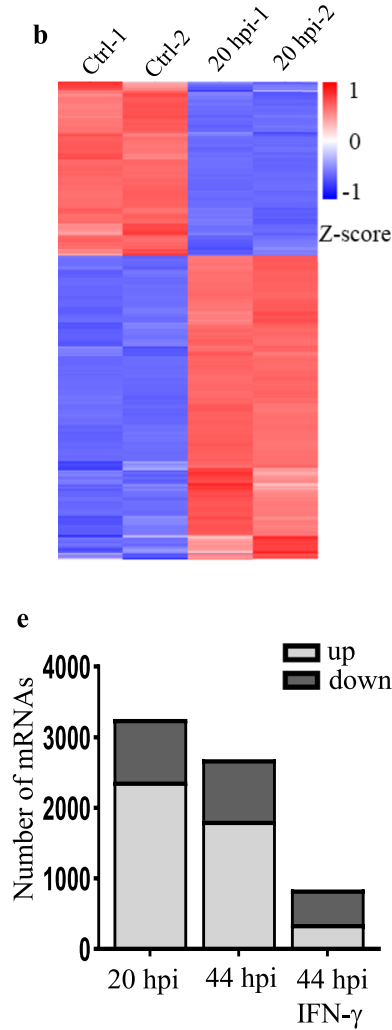

c
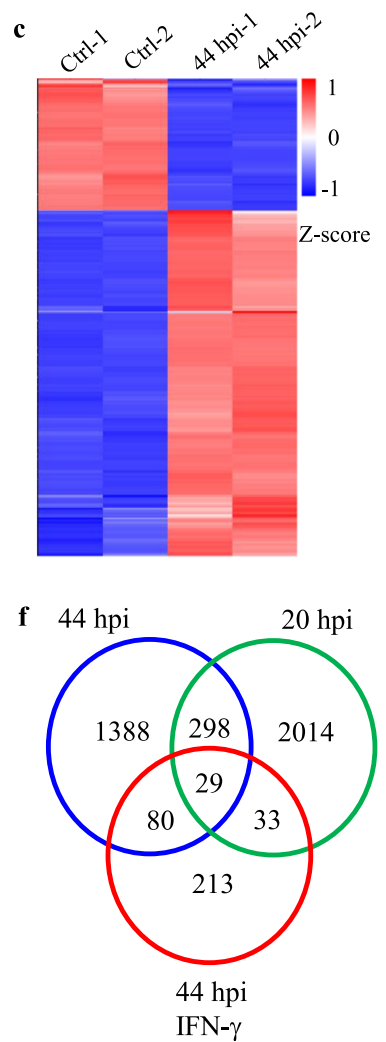

44 hpi IFN- $\gamma$
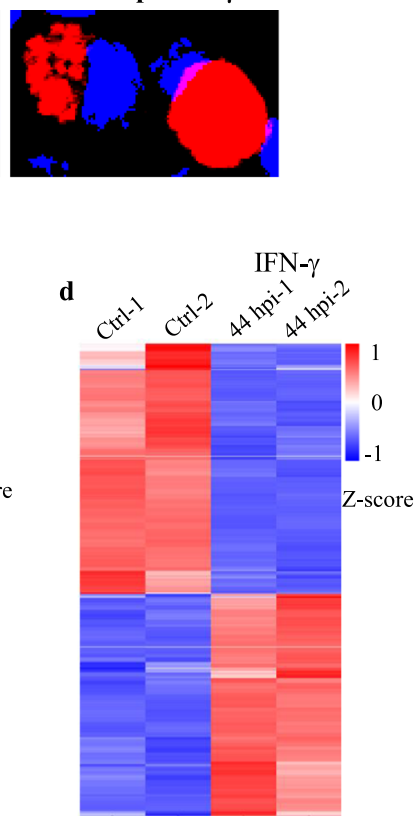

g $\quad 44 \mathrm{hpi}$ hpi

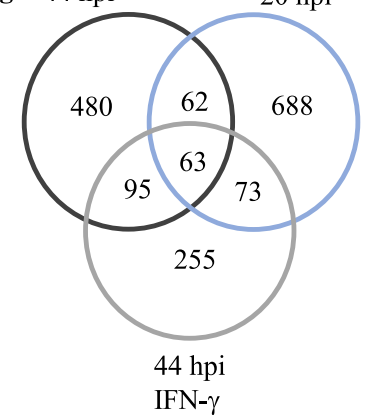

Fig. 1 Genome-wide analysis of mRNAs at different stages of C. trachomatis infection. a Microscopic images of C. trachomatis infected cells at 20 hpi, 44 hpi, and IFN- $\gamma$ treatment at 44 hpi. Red: anti-MOMP antibody, blue: DAPI. Fluorescence microscopy was carried out using Leica DM IL LED microscope (Wetzlar, Germany) and processed with Image J (https://imagej.nih.gov/ij/). Scale bar indicates $10 \mu m$. b-d Heatmap plots showing differentially expressed host cell mRNAs at $20 \mathrm{hpi}, 44 \mathrm{hpi}$, and $44 \mathrm{hpi}$ with IFN- $\gamma$ treatment as compared to their respective negative controls. Cutoff value $>1$ reads and $P$-value $<0.05$. Heatmaps were created with $\mathrm{R}$ version 3.6 .3 (https://www.r-project.org/). e The number of host cell DEGs at $20 \mathrm{hpi}, 44 \mathrm{hpi}$, and $44 \mathrm{hpi}$ with IFN- $\gamma$ treatment. f Venn diagram of upregulated mRNAs at $20 \mathrm{hpi}, 44 \mathrm{hpi}$, and $44 \mathrm{hpi}$ with IFN- $\gamma$ treatment. $\mathbf{g}$ Venn diagram downregulated mRNAs in C. trachomatis-infected host cells. The differentially expressed genes are defined as those with fold change $\geq 2$ (upregulated) or $\leq 0.5$ (downregulated) between the groups and their respective controls with an adjusted P-value $<0.05$. Data are the average expression from two independent experiments

dysregulated mRNAs occurred at 20 hpi whiles IFN- $\gamma$ treatment for 44 hpi showed the least dysregulation of coding genes during C. trachomatis infection (Fig. 1g). Taken together, these data suggest that the different stages of $C$. trachomatis infection modulate the expression of distinct sets of host genes.

Next, we analyzed the differentially expressed lncRNAs among infected cells at $20 \mathrm{hpi}, 44 \mathrm{hpi}$, and $44 \mathrm{hpi}$ with IFN- $\gamma$ treatment. At 20 hpi, we identified 3528 differentially expressed lncRNAs comprising of 3291 upregulated lncRNAs (93.3\%) and 237 downregulated lncRNAs
(6.7\%) (Fig. 2a-c, Supplementary Fig. 2a). At 44 hpi without IFN- $\gamma$ treatment, 1349 differentially expressed lncRNAs comprising of 907 upregulated lncRNAs (67.2\%) and 442 downregulated lncRNAs (32.8\%) (Fig. 2a-c, Supplementary Fig. 2b). For 44 hpi IFN- $\gamma$ treated cells, 339 differentially expressed lncRNAs (47 upregulated and 292 downregulated) were identified as compared to the controls (Fig. 2a-c, Supplementary Fig. 2c). Surprisingly, almost $86 \%$ of the overall differentially expressed lncRNAs at $44 \mathrm{hpi}$ with IFN- $\gamma$ treatment were downregulated as compared to only $7 \%$ at $20 \mathrm{hpi}$ 

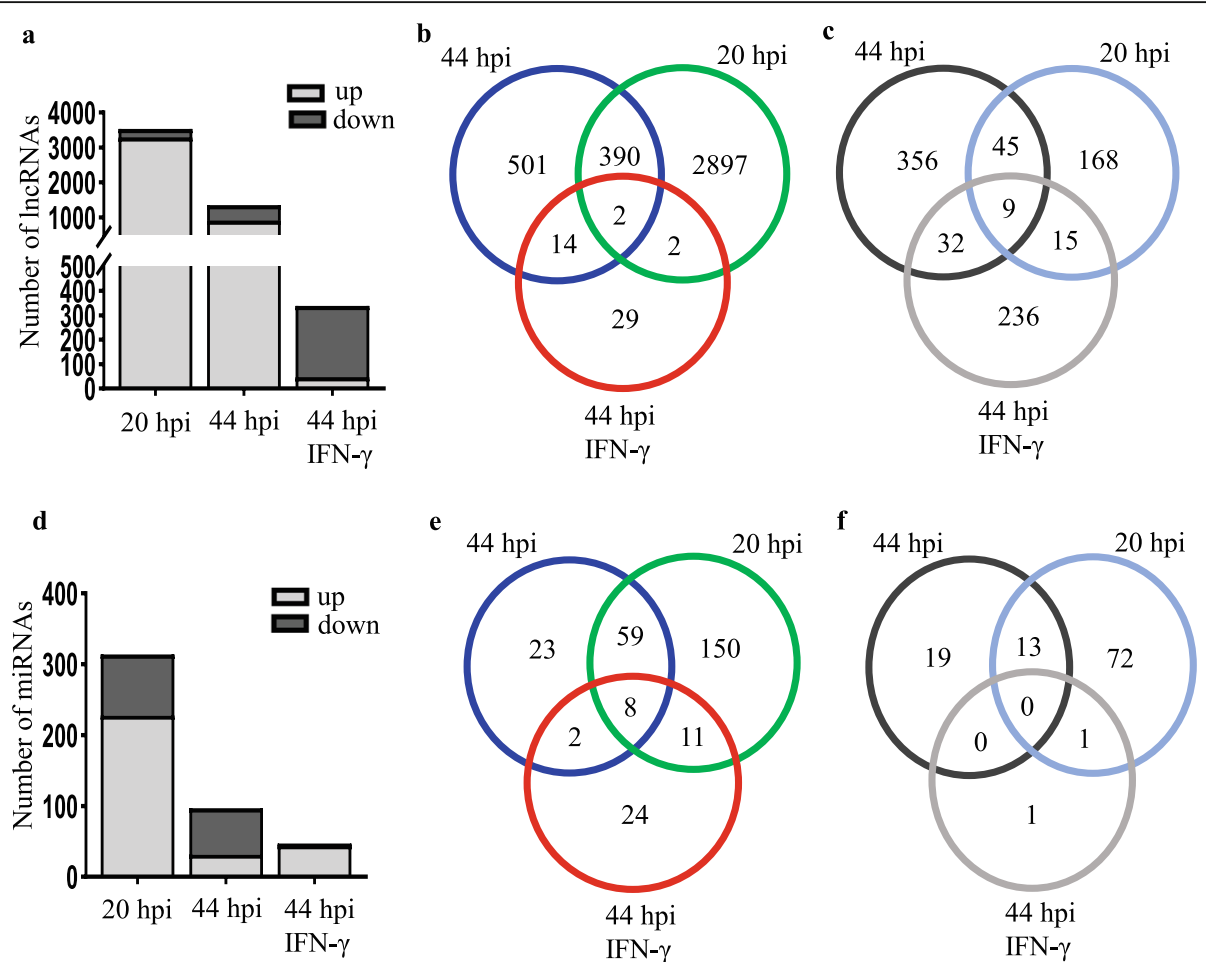

Fig. 2 Expression patterns of host cell IncRNAs and miRNAs during C. trachomatis development. a Differentially expressed IncRNAs in C. trachomatisinfected cells at 20 hpi, 44 hpi, and 44 hpi under IFN- $\gamma$ treatment. b Venn diagram of upregulated IncRNAs in C. trachomatis-infected host cells. c Venn diagram downregulated IncRNAs in C. trachomatis-infected host cells. d Differentially expressed miRNAs in C. trachomatis-infected cells at 20 hpi, 44 hpi, and 44 hpi under IFN- $y$ treatment. e Venn diagram of upregulated miRNAs in C. trachomatis-infected host cells. $\mathbf{f}$ Venn diagram downregulated miRNAs in C. trachomatis-infected host cells. The differentially expressed genes are defined as those with fold change $\geq 2$ (upregulated) or $\leq 0.5$ (downregulated) between the groups and their respective controls with an adjusted $P$-value of $<0.05$. Data are the average from two independent experiments

and $33 \%$ at 44 hpi (Fig. 2c). Among the three stages of C. trachomatis infection, 20 hpi and 44 hpi shared 390 upregulated lncRNAs as compared to $14 \operatorname{lncRNAs}$ shared by host cells at $44 \mathrm{hpi}$ without IFN- $\gamma$ and $44 \mathrm{hpi}$ with IFN- $\gamma$ treatment. Host cells at 20 hpi and IFN- $\gamma$ treatment at 44 hpi (Fig. 2b) shared two lncRNAs. For lncRNAs shared by all three treatments, we observed only 2 upregulated and 9 downregulated commonly shared lncRNAs (Fig. 2b-c). Similar to the differentially expressed mRNAs, host cells at 20 hpi showed more differentially upregulated and less downregulated lncRNAs as compared to the other two treatments. Interestingly, the majority of lncRNAs in IFN- $\gamma$-treated cells at $44 \mathrm{hpi}$ were downregulated (Fig. 2c) as compared to the host cells at 20 hpi and 44 hpi without IFN- $\gamma$ treatment in which most of the differentially expressed genes were upregulated indicating reduced transcriptional activation of host genes during persistent infection of $C$. trachomatis.

In C. trachomatis-infected host cells at $20 \mathrm{hpi}$, we observed 314 differentially expressed miRNAs (228 upregulated and 86 downregulated), whiles 124 miRNAs (92 upregulated and 32 downregulated) differentially regulated at $44 \mathrm{hpi}$ (Fig. 2d-f, Supplementary Fig. 3a-b). In the presence of IFN- $\gamma$ at $44 \mathrm{hpi}$, we observed 47 differentially expressed miRNAs with only 2 downregulated miRNAs (Fig. 2e-f, Supplementary Fig. 3c). Only 23 miRNAs were uniquely upregulated in the infected cells at $44 \mathrm{hpi}$ as compared to 150 miRNAs at $20 \mathrm{hpi}$ and 24 miRNAs in IFN- $\gamma$-treated cells at 44 hpi (Fig. 2e). Furthermore, we observed that only 8 host cell miRNAs were significantly expressed at all developmental stages of $C$. trachomatis infection (Fig. 2e-f). Surprisingly, all three treatments shared no commonly downregulated miRNAs. The highest number of differentially expressed miRNAs was observed at $20 \mathrm{hpi}$. These data suggest that during C. trachomatis infection, the different developmental stages of $C$. trachomatis infection modulate the transcriptional regulation of certain critical non-coding genes that are essential for host cell survival and may play important roles in the pathogenesis of C. trachomatis.

\section{Verification of candidate mRNAs and miRNAs specific for} different stages of $C$. trachomatis infection

Next, we validated the significantly upregulated mRNAs and miRNAs in C. trachomatis-infected host cells at specific stages of infection. Similar to the results obtained from our RNA-seq data, the top 5 upregulated mRNAs showed distinct expression levels at $20 \mathrm{hpi}, 44 \mathrm{hpi}$, and 
44 hpi under IFN- $\gamma$ treatment using a quantitative RTqPCR. The expression levels of ENAM, SYT5, ANKR DF20, ACTC1, and CCDC144NL were predominantly upregulated at $20 \mathrm{hpi}$, but not at $44 \mathrm{hpi}$ with or without IFN- $\gamma$ treatment (Fig. 3a). We found that the expression levels of FGF21, PLA2G4B, MIOX, INHBE, and ADM2 significantly increased at $44 \mathrm{hpi}$ but not at $20 \mathrm{hpi}$ or 44 hpi with IFN- $\gamma$ treatment (Fig. 3b). Similarly, after IFN- $\gamma$ treatment for $44 \mathrm{hpi}$, all the top 5 upregulated host genes (NUDT4B, MPZ, OASL, IFI44, and INP P4B) showed significant expression levels exclusively in the IFN- $\gamma$ treated host cells as compared to host cells harboring C. trachomatis at $20 \mathrm{hpi}$ or $44 \mathrm{hpi}$ without IFN- $\gamma$ treatment (Fig. 3c).

Furthermore, the top 5 miRNAs identified from the miR-seq data were also validated. At $20 \mathrm{hpi}$, miRNAs such as miR-137-3p, miR-370-3p, miR-487a-5p, miR708-5p, and miR-1185-5p were significantly upregulated (Fig. 4a) while miR-4474-3p, miR-6529-5p, miR-68275p, miR-6834-5p, and miR-6879-3p were dramatically upregulated in C. trachomatis-infected host cells at 44 hpi (Fig. 4b). Among the miRNAs expressed only in the IFN- $\gamma$-treated cells at $44 \mathrm{hpi}, \mathrm{miR}-1-3 \mathrm{p}, \mathrm{miR}-142-3 \mathrm{p}$, miR-150-5p, and miR-363-3p were significantly expressed only at $44 \mathrm{hpi}$ in the presence of IFN- $\gamma$. miR133a-3p showed upregulation at all 3 stages of C. trachomatis infection (Fig. 4c). Our results confirmed the hypothesis that $C$. trachomatis infection modulate specific gene expressions at the different stages of $C$. trachomatis infection in host cells.

\section{miR-193b as a potential serum biomarker of $C$.}

\section{trachomatis infection}

We also tested 5 out of the 8 commonly upregulated miRNAs at $20 \mathrm{hpi}$, 44 hpi with or without IFN- $\gamma$ treatment (Fig. 2e), and found that 3 miRNAs (miR-139-3p, miR-193b-5p, and miR-365a-5p) were significantly expressed in C. trachomatis-infected host cells (Fig. 5a). Interestingly, the expression levels of miR-139-3p and miR-193b-5p were significantly higher in the serum samples of C. trachomatis-infected patients as compared to the sera from healthy blood donors. To determine whether the two miRNAs could differentiate between active and past $C$. trachomatis infections, we tested serum samples from patients who were previously diagnosed by nucleic acid test (NAT) or antibody test for C. trachomatis. Both miR-139-3p and miR-193b-5p showed increased expression levels regardless of active $C$. trachomatis infection $(\mathrm{NAT}+/ \mathrm{Ab}+)$ or past infection (NAT-/Ab+) (Fig. 5b-c).

Furthermore, we asked whether these miRNAs are specifically expressed only during $C$. trachomatis infection. Hence, we measured miR-139-3p and miR-193b-5p expression levels in serum samples of previously diagnosed HIV-1-infected patients. We found that miR139-3p was significantly expressed in HIV-1-infected serum samples whereas miR-193b-5p showed no significant expression in HIV-1-infected serum samples as compared to the serum samples from healthy individuals (Fig. 5b-c). Taken together, these results suggest the involvement of $C$. trachomatis infection in the perturbation of the host genomes leading to the expression of specific miRNAs that may play important roles in maintaining pathogen infection and development. Also, the detection of circulating miRNAs that are specifically expressed in the sera during C. trachomatis infection, such as miR-193b-5p, may find potential application in the clinical diagnosis of $C$. trachomatis infection.

To identify the potential target genes of the commonly upregulated host cell miRNAs during $C$. trachomatis infection, we performed competing endogenous RNAs network analysis of significantly upregulated miRNAs at $20 \mathrm{hpi}$ and also at $44 \mathrm{hpi}$ with without IFN- $\gamma$ treatment and their corresponding downregulated mRNAs. The potential miRNA target genes were predicted with TargetScanHuman7.2 software. Among the commonly upregulated miRNAs at $20 \mathrm{hpi}$ and at $44 \mathrm{hpi}$ with without IFN- $\gamma$ treatment, miR-193b-5p showed the highest degree of interaction with downregulated mRNAs at 20 hpi (Fig. 5d).

\section{Identification of stage-specific pathways in host cells}

We then performed gene ontology (GO) annotation and Kyoto Encyclopedia of Genes and Genomes (KEGG) pathway analysis of the differentially upregulated genes during $C$. trachomatis infection. Interestingly, we observed that the differentially upregulated genes at various stages of $C$. trachomatis infection regulate distinct signaling pathways in the host cells (Fig. 6a). Comparison between the biological processes of the host genes at the various stages of $C$. trachomatis infection showed that the host cells at 20 hpi showed the most significantly regulated biological processes such as the regulation of cell adhesion, extracellular matrix organization, and cellsubstrate adhesion. Differentially upregulated genes at $44 \mathrm{hpi}$ with IFN- $\gamma$ treatment were enriched for cytokinemediated signaling pathways, chaperone-mediated protein folding, and cellular response to IFN- $\gamma$. At $44 \mathrm{hpi}$, host cells exhibited the least regulation of biological process and function (Fig. 6a). Detailed analysis of these pathways revealed that at $44 \mathrm{hpi}$, the differentially upregulated genes were enriched for the $\mathrm{T}$ cell signaling, mTOR signaling, and Rap1 signaling pathways. However, at $20 \mathrm{hpi}$, most differentially upregulated host genes were essentially involved in antigen processing and presentation. Under IFN- $\gamma$ treatment at $44 \mathrm{hpi}$, the host genes were mainly enriched for cytokine-cytokine 
a

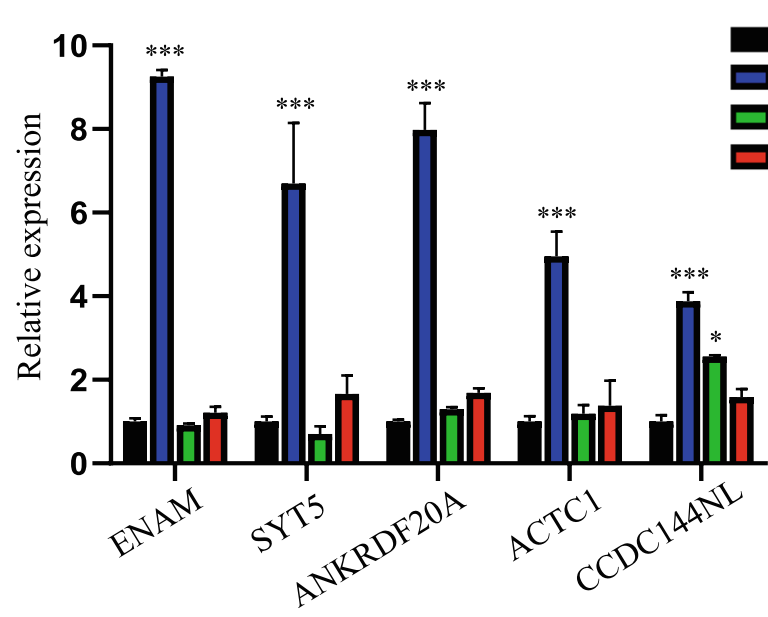

b

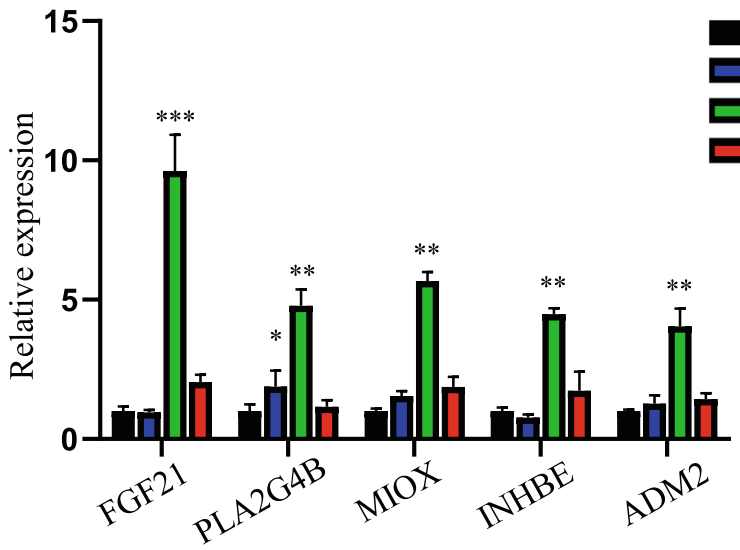

c

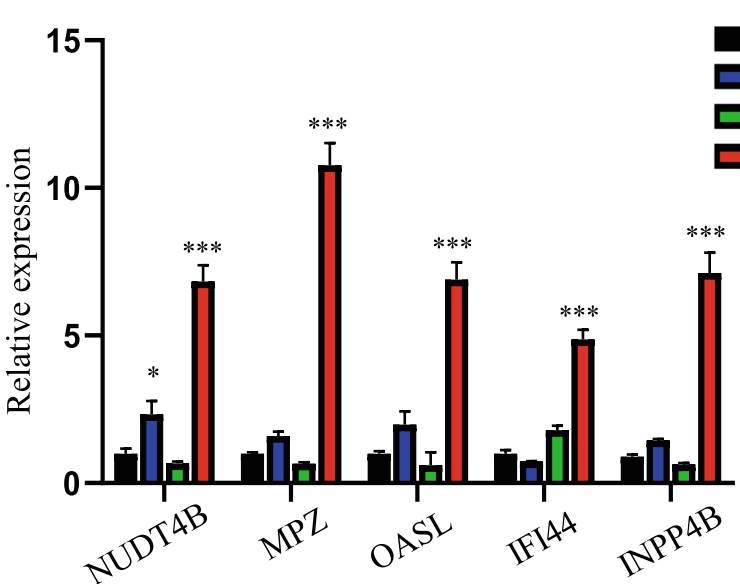

Fig. 3 Top 5 most differentially expressed mRNAs in C. trachomatis-infected host cells. Relative expression levels of mRNAs at 20 hpi (a), 44 hpi without IFN- $\gamma$ treatment $(\mathbf{b})$, and 44 hpi with IFN- $\gamma$ treatment $(\mathbf{c})$. Data are mean \pm SEM from three independent experiments with three replicates. Student's t-test, ${ }^{*} P<0.05$, ${ }^{* *} P<0.01,{ }^{* * *} P<0.001$

receptor interaction, FoxO signaling, and Ras signaling pathways (Fig. 6b). Taken together, our analyses showed that different stages of $C$. trachomatis infection modulate distinct host cell signaling pathways and may play critical roles in modulating $C$. trachomatis infection and the survival of the host cells.

\section{Discussion}

With the increasing incidence of $C$. trachomatis infection worldwide, many countries have now considered it a major public health concern. C. trachomatis infection results in innate and adaptive immune responses that enable the host cells to attack, destroy, and clear the 


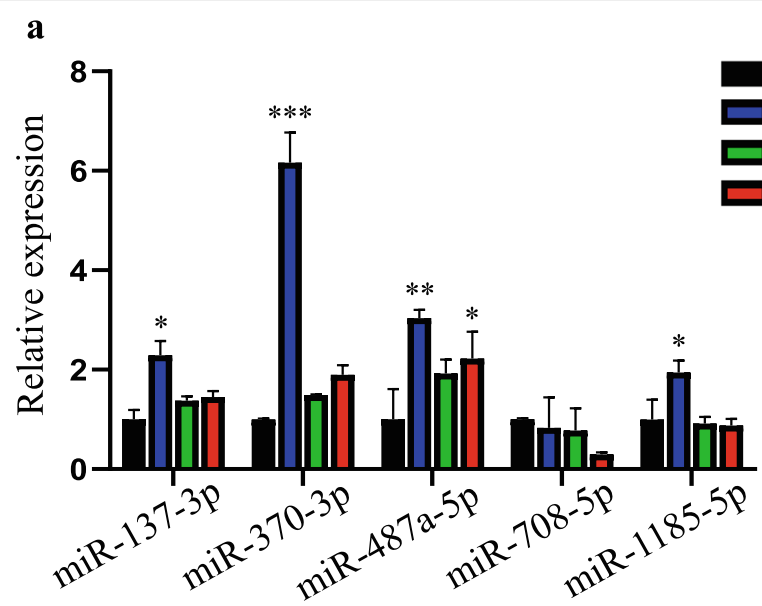

b
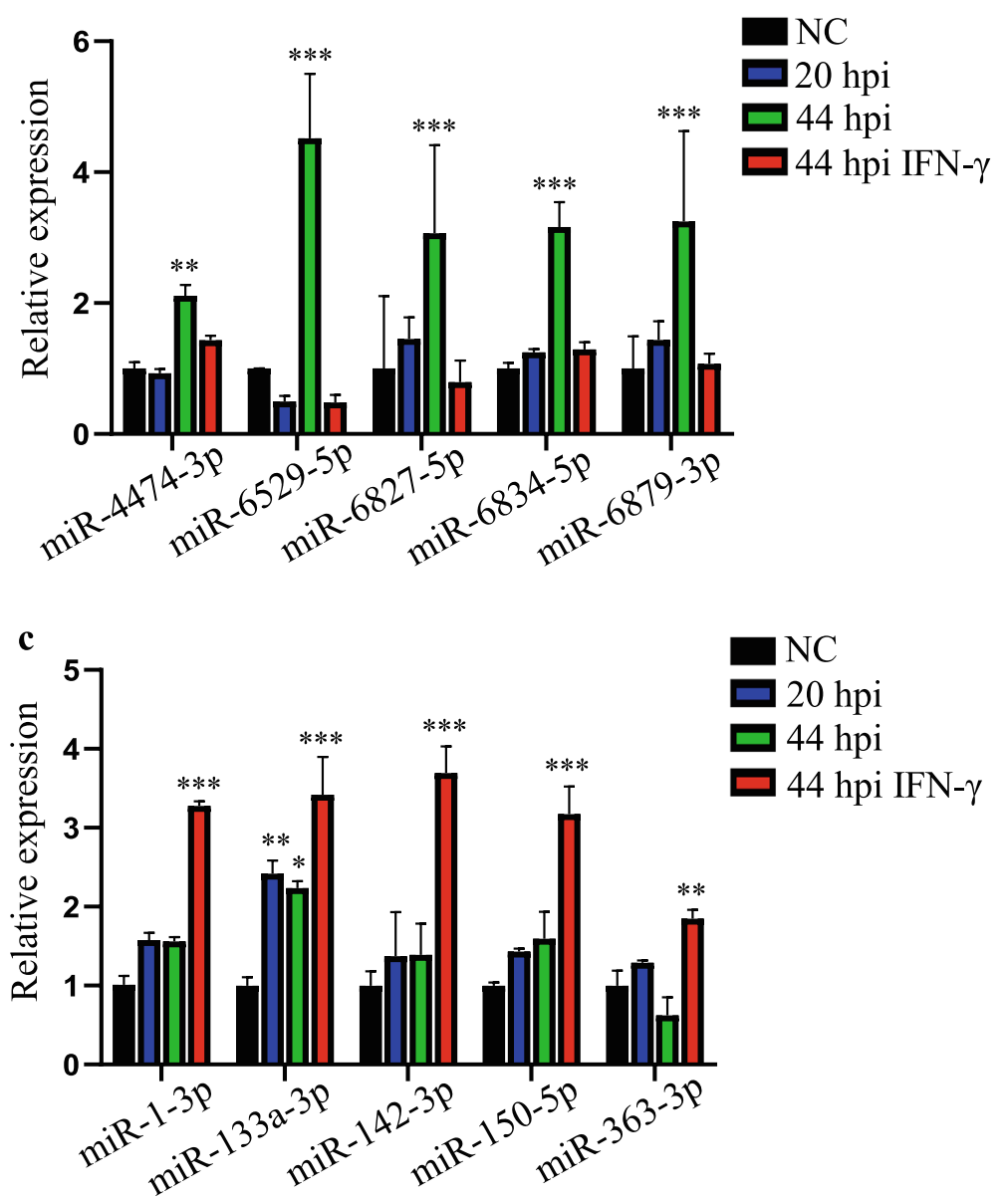

Fig. 4 Expression levels of host cell miRNAs during C. trachomatis-infection. RT-qPCR analysis of the relative expression levels of the top 5 miRNAs at 20 hpi (a), 44 hpi without IFN- $\gamma$ treatment (b), and 44 hpi with IFN- $\gamma$ treatment (c). Data are mean \pm SEM from three independent experiments with three replicates. Student's t-test, ${ }^{*} P<0.05,{ }^{* *} P<0.01,{ }^{* * *} P<0.001$

bacteria [6]. C. trachomatis must undergo its biphasic developmental cycle involving an infectious EB and a replicative form $\mathrm{RB}$ during the infection of the host cells $[5,6]$. Under unfavorable conditions, such as the presence of antibiotics or IFN- $\gamma$ treatment, C. trachomatis may enter the persistent ARB form to enable it to survive. These developmental processes require the synergistic interaction between the host and bacteria 
a

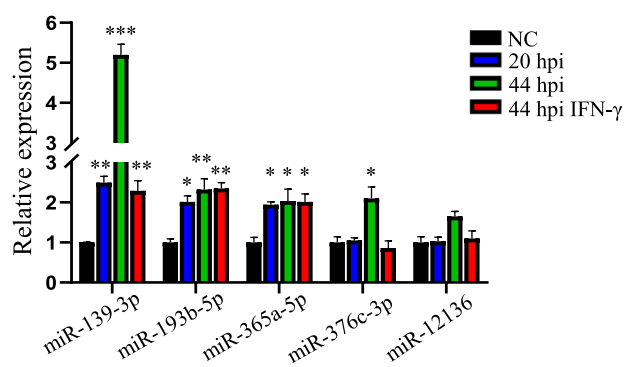

b
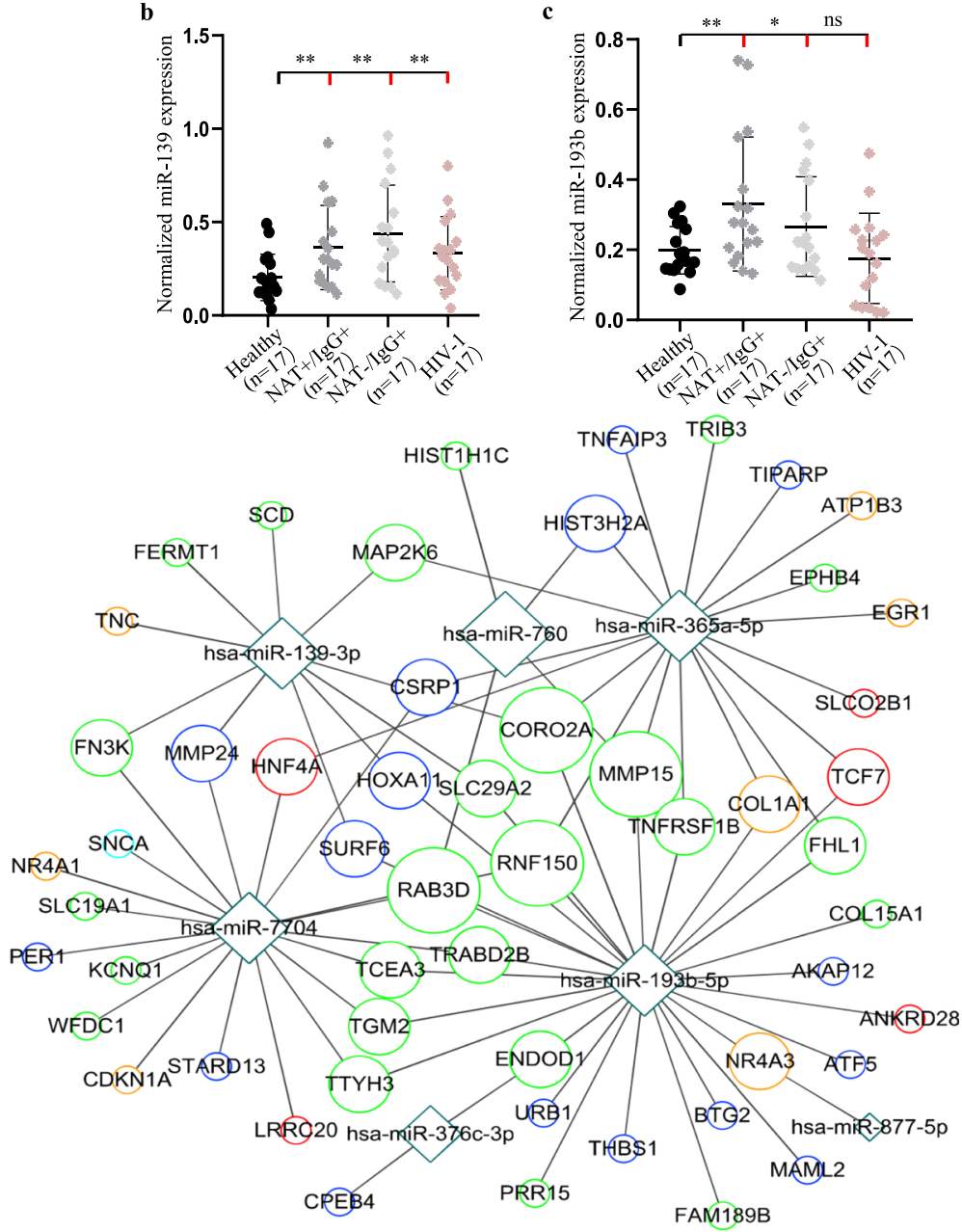

Fig. 5 Analysis of commonly expressed miRNAs post-C. trachomatis infection. a Relative expression levels of the top 5 miRNAs that were commonly expressed at all stages of C. trachomatis infection. Normalized expression levels of miR-139-3p (b) and miR-193b-5p (c) in serum samples from C. trachomatis patients, HIV-1 patients, and healthy controls. NAT+/Ab+ indicates positive for nucleic acid and C. trachomatis antibody. NAT-/Ab+ indicates negative for nucleic acid, but positive for $C$. trachomatis antibody. All serum samples were recovered from - $80{ }^{\circ} \mathrm{C}$ after almost a year of storage. Data are mean \pm SEM of three replicates for each serum sample $(n=17)$. Relative expressions were calculated using the delta-delta Ct method after normalization with the expression levels of GAPDH in each sample. Student's t-test, ${ }^{*} P<0.05,{ }^{* *} P<0.01$, ${ }^{* * *} P<$ 0.001 by comparing healthy samples (black) with $C$. trachomatis or HIV-1-infected serum samples (red). Images (a, b, c) were created and analyzed with GraphPad Prism 8 (https://www.graphpad.com/scientific-software/prism/). d mRNA-miRNA interaction network between the top 5 commonly upregulated miRNAs $20 \mathrm{hpi}, 44$, and $44 \mathrm{hpi}$ with IFN- $\gamma$ treatment and their potential targets. The potential miRNA target genes were predicted with TargetScanHuman7.2 software (http://www.targetscan.org/vert_72/). The network was drawn with Cytoscape version 3.0.1 (http:// www.cytoscape.org/). Circles indicate the target host genes whereas the squares indicate the miRNAs. The size of the circle represents the degree of the node adjusted according to the number of targeted miRNAs. The color of the circles represents the stage of $C$. trachomatis infection: green, 20 hpi; blue, 44 hpi; red, 44 hpi with IFN- $\gamma$ treatment; orange, both 20 hpi and 44 hpi with IFN- $\gamma$; light blue, 44 hpi with or without IFN- $\gamma$ 
genomes $[21,22]$. Hence, different stages of C. trachomatis infection may require the transcription and posttranscriptional regulation of specific sets of host genes.

RNA sequencing data from host cells infected with $C$. trachomatis indicate that $C$. trachomatis infection modulates dynamic gene expression shift across the genome of the host cells at specific time points during the development cycle of C. trachomatis. C. trachomatis-infected host cells at 20 hpi showed the most significant host gene dysregulation as compared to 44 hpi with or without IFN- $\gamma$ treatment (Fig. 1, Fig. 2). Among the dysregulated host genes, we observed 2014, 1388, and 213 mRNAs that were exclusively upregulated $20 \mathrm{hpi}, 44 \mathrm{hpi}$ without IFN- $\gamma$, and $44 \mathrm{hpi}$ with IFN- $\gamma$ treatment, respectively, and only 29 commonly regulated genes in all three treatments (Fig. 1). A similar trend was observed for differentially expressed lncRNAs and miRNAs of the host cells at $20 \mathrm{hpi}, 44 \mathrm{hpi}$ without IFN- $\gamma$, and $44 \mathrm{hpi}$ with IFN- $\gamma$ treatment suggesting that different stages of C. trachomatis infection result in the modulation of a specific set of genes. Generally, a large proportion of mRNAs and lncRNAs were upregulated at $20 \mathrm{hpi}$ whereas C. trachomatis infection undergoes active replication into either the infectious or the persistent form. At 44 hpi under IFN- $\gamma$ treatment, there were very few dysregulated mRNAs, lncRNAs, and miRNAs with the majority of them showing downregulation (Fig. 1d, Fig. 2). These data show that during an active infection, the host cell responds by modifying its transcriptional regulation of genes that are crucial for its survival. However, in the host cells containing the persistent ARB forms of C. trachomatis at $44 \mathrm{hpi}$ under IFN- $\gamma$ treatment, the transcription of a large portion of the host genomes was switched off or at lower levels. These transcriptional switches may probably be due to changes in signaling pathways that are needed for the survival of the pathogen or the clearance of the pathogen at the different stages of infection [23]. Also, the persistent forms of $C$. trachomatis are less active, thereby requiring little nutrient or energy utilization from the host cells.

Circulating miRNAs have been suggested as potential biomarkers of some infectious diseases [24]. Serum miRNAs are relatively stable at variable $\mathrm{pH}$ conditions and are highly resistant to enzymatic degradation $[25,26]$. These properties make serum miRNAs suitable biomarkers for diagnosis. miR-193b-5p, which was significantly expressed at $20 \mathrm{hpi}$, and $44 \mathrm{hpi}$ with or without IFN- $\gamma$ treatment, showed increased expression in the serum of C. trachomatis-infected patients only. This observation suggests that miR-193b may serve as a potential serum biomarker for $C$. trachomatis infection (Fig. 5b-c). However, this may require further validation and analysis among a large population of C. trachomatis-infected serum samples in future investigations.
Generally, mRNA-miRNA interactions play crucial roles in the modulation of various metabolic pathways in the cells. The importance of several lncRNAs and miRNAs in transcriptional and post-transcriptional regulation of host gene expression has previously been demonstrated [27-29]. Only 8 miRNAs were often upregulated at $20 \mathrm{hpi}$ and $44 \mathrm{hpi}$ with or without IFN- $\gamma$ treatment (Fig. 2e). However, most miRNAs were upregulated at $20 \mathrm{hpi}$. Our results are consistent with the fact that active replication of $C$. trachomatis occurs within the first 20-24h of C. trachomatis infection [6].

Further analysis of the differentially upregulated genes showed enrichment of distinct signaling pathways at the specific stages of $C$. trachomatis infection. There was little overlap in the pathways regulated by differentially upregulated host genes at $20 \mathrm{hpi}, 44 \mathrm{hpi}$, and $44 \mathrm{hpi}$ with IFN- $\gamma$ treatment (Fig. 6). Host cells at 20 hpi revealed the dysregulation of biological processes such as cell adhesion, extracellular matrix organization, and cellsubstrate adhesion as previously reported [30]. The most significantly regulated pathways in cells at $44 \mathrm{hpi}$ under IFN- $\gamma$ treatment were cytokine-mediated signaling pathway and chaperone-mediated protein folding (Fig. 6). The upregulation of these pathways in host cells infected with the persistent forms of $C$. trachomatis may serve as immune surveillance or manipulation of the host immune system to allow the pathogen to escape [31].

Previous reports have shown that $C$. trachomatis infection leads to the activation of the ERK MAPK, PI3K, and RAS signaling pathways, which are major regulatory pathways in the nutrient cycle in bacteria infections [32, 33]. Also, chlamydial infection interferes with the immune response of the host cells to viral infections and the subsequent fate of the cells [33]. Detailed analysis revealed that the differentially upregulated host genes at 20 hpi were enriched for antigen processing and presentation while at $44 \mathrm{hpi}$, host genes were enriched for $\mathrm{T}$ cell receptor signaling, mTOR signaling, and Rap1 signaling pathways. In the presence of IFN- $\gamma$, the most significantly activated signaling pathways in host cells were cytokine-cytokine receptor interaction, FoxO signaling, and Ras signaling pathways (Fig. $6 \mathrm{~b}$ ), as previously reported [33]. Thus, at the various stages of Chlamydia development, there is the modulation of different components of essential host cell signaling pathways that support the survival of the pathogen [33]. Hence, a transcriptional manipulation of completely different sets of host genes and pathways at the various stages of C. trachomatis infection may be important in the survival of the host cells as the complexity and content of the inclusion body changes [23]. The activation of these essential signaling pathways in the host cells at the various stages of $C$. trachomatis infection suggests that $C$. trachomatis induces a combination of pathways that are responsible 


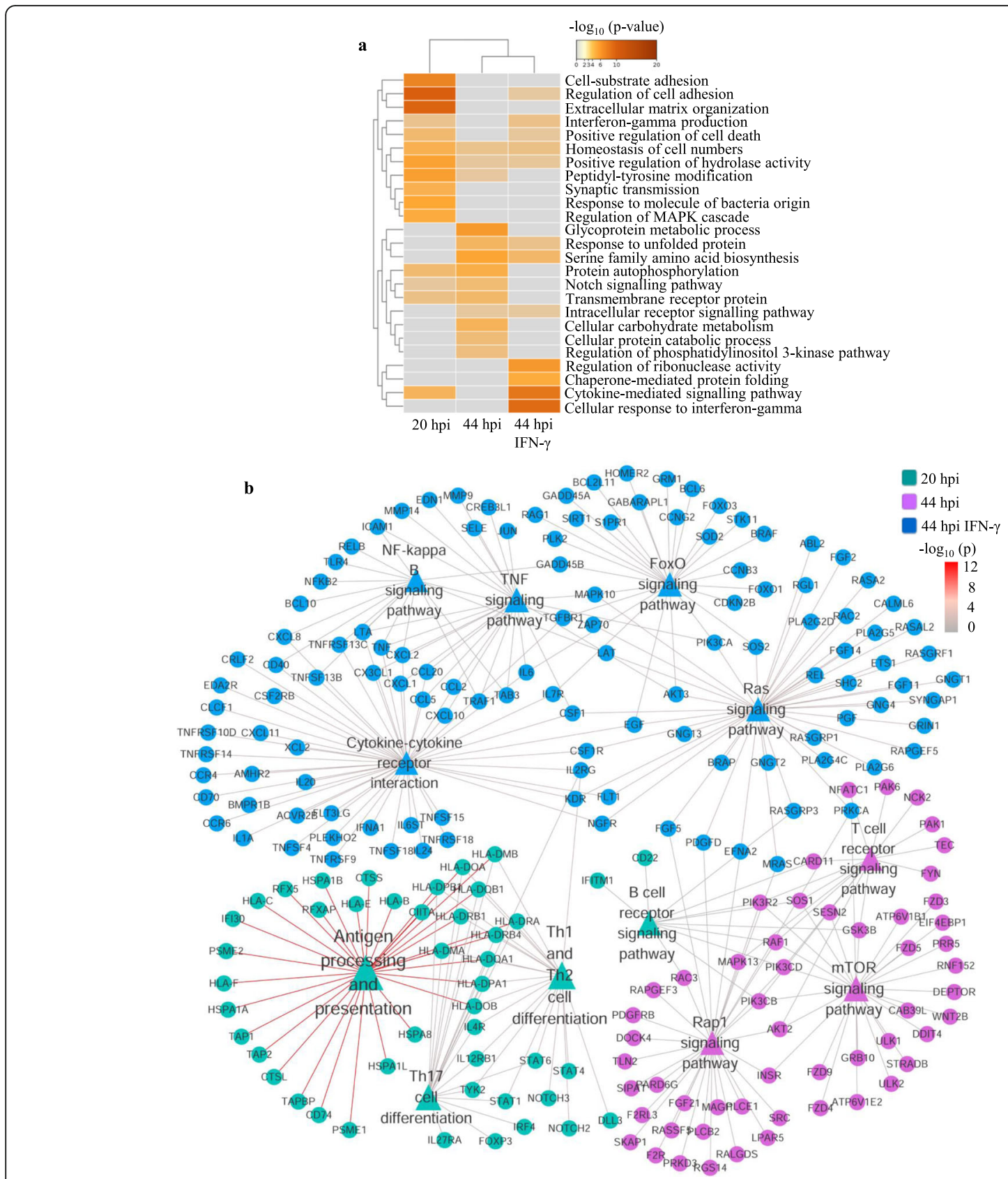

Fig. 6 Gene ontology (a) and KEGG (b) pathways for differentially upregulated host cell mRNAs during C. trachomatis infection. Data represent the top 25 biological processes at 20 hpi, 44 hpi, and 44 hpi with IFN- $\gamma$. The color of the connecting line is representative of the $p$-value. Images were created with FunRich 3.1.3 (http://funrich.org/)

for the survival of the host cells. The specific role of the differentially regulated host genes at each developmental stage of $C$. trachomatis-infection remains to be investigated.

\section{Conclusions}

Our current study demonstrated that $C$. trachomatis infection of HeLa cells modulates the expression levels of mRNAs, lncRNAs, and miRNAs, which shows specificity for different stages of $C$. trachomatis infection and may influence the host cell response to infection. The dysregulation of these stage-specific host genes results in the differential regulation of important signaling pathways that are necessary for the survival of the pathogen. Serum expression of miR-193b could serve as a potential serum biomarker in the diagnosis of $C$. trachomatis infection for improved management and control of the disease. RNA-seq and miR-seq data obtained from this 
study could also serve as an essential resource for future investigations into the molecular mechanisms at specific developmental stages of C. trachomatis.

\section{Methods}

\section{Cells, bacteria, and clinical samples}

HeLa229 cells were purchased from American Type Culture Collection (Manassas, VA, USA) and cultured in Dulbecco's modified Eagle's medium (DMEM) supplemented with $10 \%$ heat-inactivated fetal bovine serum (Sigma-Aldrich, USA) at $37{ }^{\circ} \mathrm{C}$ with $5 \% \mathrm{CO}_{2}$. C. trachomatis serovar $\mathrm{E}$ was a kind gift from the Clinical Microbiology Laboratory of the Chenzhou No. 1 People's Hospital (Chenzhou, China) and was routinely preserved in our laboratory.

\section{Infection of HeLa cells}

HeLa cells were infected with C. trachomatis as previously described [34] with little modification. Briefly, $1 \times$ $10^{5} \mathrm{HeLa}$ cells in 6-well plates were cultured overnight. Cells were first treated with DEAE-Dextran at $30 \mathrm{mg} / \mathrm{L}$ before infection with $C$. trachomatis at a multiplicity of infection (MOI) of 1 by centrifugation onto the host cells at $1000 \mathrm{rpm}$ for $1 \mathrm{~h}$. The MOI was calculated using the inclusion-forming unit (IFU) with $\mathrm{MOI}=\mathrm{IFU} /$ number of cells. EBs were purified and preserved in a sucrose-phosphate-glutamate buffer before storage at $80^{\circ} \mathrm{C}$. For RNA extraction, C. trachomatis-infected host cells at the various stages of $C$. trachomatis infection were collected as follows: $20 \mathrm{hpi}, 44 \mathrm{hpi}$, and $44 \mathrm{hpi}$ with IFN-y (50 units) treatment immediately after infection. Uninfected HeLa cells were used as respective negative controls at 20 and $44 \mathrm{hpi}$, and IFN- $\gamma$-treated cells were used as negative controls for 44 hpi IFN- $\gamma$-treated $C$. trachomatis-infected cells.

\section{Immunofluorescence assay}

Cells were washed twice with PBS followed by incubation with $4 \%$ formaldehyde at room temperature for 30 mins. After a single wash, cells were then incubated with $0.1 \%$ Triton X-100 at room temperature for 10 mins and washing three times with PBS. Wells were blocked with $4 \%$ bovine serum albumin (BSA) for $1 \mathrm{~h}$ followed by incubation with $2 \mu \mathrm{g} / \mathrm{ml}$ anti-MOMP antibody (Abcam, Cambridge, UK) and 1:1000 dilution of DAPI (Sigma, USA) at $37^{\circ} \mathrm{C}$ for $1 \mathrm{~h}$. Cells were washed thrice with PBS and then incubated with $2 \mu \mathrm{g} / \mathrm{ml}$ CoraLite 594 fluorescence anti-mouse IgG (Proteintech, USA) for 30 mins at $37^{\circ} \mathrm{C}$. Cells were observed after washing three times with PBS. Images were obtained with.

\section{RNA extraction}

Total RNAs from C. trachomatis-infected cells were extracted using TRIzol L/S (Invitrogen, USA) according to the manufacturer's instructions with few modifications, as described previously [29]. Serum miRNAs were extracted using the miRNeasy Serum/Plasma Kit (Qiagen Inc., Germany) according to the manufacturer's instruction. Briefly, frozen serum samples were thawed on ice and $200 \mu \mathrm{l}$ of each serum sample was mixed thoroughly with $1 \mathrm{ml}$ QIAzol lysis reagent. Chloroform $(200 \mu \mathrm{l})$ was added and incubated at room temperature for $5 \mathrm{~min}$. Tubes were centrifuged for $15 \mathrm{~min}$ at $12,000 \times \mathrm{g}$ at $4{ }^{\circ} \mathrm{C}$. The aqueous phase was then mixed with $900 \mathrm{ml}$ of $100 \%$ ethanol. About $700 \mu \mathrm{l}$ of the mixture was loaded into the RNeasy minElute spin column in a $2 \mathrm{ml}$ collection tube and followed by centrifugation. The column was then washed with $700 \mu \mathrm{l}$ of Buffer RWT, $500 \mu \mathrm{l}$ of Buffer RPE, and $500 \mu \mathrm{l}$ of $80 \%$ ethanol. RNA was eluted with $14 \mu \mathrm{l}$ RNase-free water.

\section{RNA sequencing and analysis}

For next-generation RNA sequencing, sequencing libraries were constructed as previously described with modifications [35] and sequencing was carried out with the BGISEQ-500 system at the Beijing Genome Institute (Wuhan, China). The quality of the raw sequence reads was assessed using FastQC [36]. Sequenced reads were trimmed for adaptor sequence, and masked for lowcomplexity or low-quality sequences, then mapped to hg38 whole genome using bowtie v2.2.5 with parameters -q --phred64 --sensitive --dpad 0 --gbar 99,999,999 --mp 1, 1 --np 1 --score-min L,0,-0.1 -I 1 -X 1000 --no-mixed --no-discordant -p 1 -k 200, and RSEM v1.2.12 with parameters --forward-prob 0. Raw data were processed using the Zebra call and CASAVA pipeline softwares. For miRNA-seq, the data were mapped to the hy38 whole genome and the miRNA database in miRBase with bowtie $(-\mathrm{v} 1)$. Data normalization and differential analysis were done using the default methods in the DESeq $R$ package [37]. The read counts of samples were normalized for sequencing depth and distortion caused by highly differentially expressed genes. A negative binomial model was used to test the significance of differential expression between the two conditions. A cutoff of False Discovery Rate (FDR) of less than 0.05 and a log fold change $>2.0$ was used to select the significant DEGs by computing the $p$-values.

\section{Quantitative RT-PCR analysis}

First-strand cDNA was synthesized with $500 \mathrm{ng}$ of total RNA using TransScript All-in-One First-Strand cDNA Synthesis SuperMix (Transgen Biotech, China). For mRNA validation, primers spanning 70-200 bp of the mRNA sequence were designed and used in the validation. MiRNA specific RTprimers were used for first-strand cDNA synthesis of $500 \mathrm{ng}$ of serum-extracted miRNA followed by the use of universal miRNA reverse primer and miRNA specific forward primers 
for miRNA validation. For RT-qPCR, the SYBR Green qPCR Master Mix (Accurate Biotechnology, China) with cycling conditions of $95^{\circ} \mathrm{C}$ for $5 \mathrm{~min}$ followed by 50 cycles of $95^{\circ} \mathrm{C}$ for $10 \mathrm{~s}, 60^{\circ} \mathrm{C}$ for $10 \mathrm{~s}$, and $72{ }^{\circ} \mathrm{C}$ for $10 \mathrm{~s}$ in the LightCycler 96 SW 1.1 real-time PCR system (Roche, USA) was used according to standard procedures. GAPDH was used as control. A list of primers is provided in Supplementary Table 1.

Gene ontology (GO) annotation and Kyoto Encyclopedia of Genes and Genomes (KEGG) pathway enrichment analysis.

GO functional annotation was carried out using the Gorilla web-server [38]. The Enrichr database (http:// amp.pharm.mssm.edu/Enrichr/) was utilized KEGG pathway enrichment analysis of upregulated mRNAs [39]. $P$-values were calculated with default parameters.

\section{Statistical analysis}

All RNA-seq data are from two biological replicates. Quantitative data were analyzed using GraphPad Prism from three independent experiments. The miRNAmRNA network was illustrated with Cytoscape [40].

\section{Supplementary Information}

The online version contains supplementary material available at https://doi. org/10.1186/s12866-020-02061-6.

\begin{abstract}
Additional file 1: Supplementary Fig. 1 Differentially expressed mRNAs in C. trachomatis-infected HeLa cells at 20 hpi (a), 44 hpi (b), and 44 hpi with IFN- $\gamma$ treatment (c). The cutoff criteria used in the volcano plot were a fold-change of 2 with a $P$-value of $<0.05$. $\log _{2}$ of average reads from two biological replicates were plotted. The heatmap plot shows the upregulated host gene expression with cutoff value $>1$ and $P_{-}$
\end{abstract} value $<0.05$

Additional file 2: Supplementary Fig. 2 Scatter plot of IncRNAs in cells containing C. trachomatis at $20 \mathrm{hpi}$ (a), $44 \mathrm{hpi}$ (b), and $44 \mathrm{hpi}$ with IFN- $\gamma$ treatment (c). The criteria used were a fold-change of $\geq 2$ or $\leq-2$, and a P-value of $<0.05$. $\log _{2}$ of average reads from two biological replicates were plotted

Additional file 3: Supplementary Fig. 3 Scatter plot of miRNAs in cells containing C. trachomatis at 20 hpi (a), 44 hpi (b), and 44 hpi with IFN- $\gamma$ treatment (c). The criteria used were a fold-change of $\geq 2$ or $\leq-2$, and a $\mathrm{P}$-value $<0.05$. $\log _{2}$ of average reads from two biological replicates were plotted.

Additional file 4: Supplementary Table 1 List of Primers used in this study.

\section{Acknowledgments}

The authors thank the Beijing Genome Institute for their support in RNA-seq and miR-seq and analysis.

\section{Authors' contributions}

EED and ST conceived and designed the experiments. EED, LH, HC, JS, and YX performed the experiments. EED, LH, SW, XW, FK, HZ, and BY performed the data analysis. EED, FR, and ST wrote the manuscript. All authors read and approved the final manuscript. All authors have read and agreed to the published version of the manuscript.

\section{Funding}

The China Postdoctoral Science Foundation (F119PF0002), the postdoctoral grant of the Dermatology Hospital of Southern Medical University (No. 2018), and the Bureau of Science and Information Technology of Guangzhou Municipality (No. 201704020219) supported this work.
Availability of data and materials

RNA-seq/miR-seq data generated in this study have been deposited in NCBI's Gene Expression Omnibus and are accessible through GEO accession number GSE158814.

\section{Ethics approval and consent to participate}

Informed consent was obtained from all subjects or if subjects are under 18, from a parent and/or legal guardian. All the experiments were carried out by following the relevant guidelines and regulations (Declaration of Helsinki). Serum samples used in this study were from previously diagnosed C. trachomatis- or HIV-1-infected patients, and the study was approved by the institutional review board protocols of the Dermatology Hospital Southern Medical University (2018015).

\section{Consent for publication}

Not applicable.

\section{Competing interests}

The authors have no conflict of interest to declare.

\section{Author details}

'Dermatology Hospital of Southern Medical University, Guangzhou, China. ${ }^{2}$ Department of Molecular Biology and Biotechnology, School of Biological Sciences, College of Agriculture and Natural Sciences, University of Cape Coast, Cape Coast, Ghana. ${ }^{3}$ Guangdong Provincial Key Laboratory of Tropical Disease Research, School of Public Health, Southern Medical University, Guangzhou, China. ${ }^{4}$ Hefei National Laboratory for Physical Sciences at Microscale, the CAS Key Laboratory of Innate Immunity and Chronic Disease, School of Life Sciences, University of Science and Technology of China, Hefei, China. ${ }^{5}$ The First People's Hospital of Chenzhou, University of South China, Chenzhou, Hunan, China.

Received: 9 September 2020 Accepted: 24 November 2020 Published online: 04 January 2021

\section{References}

1. World Health Organization. Sexually transmitted infections (STIS), fact sheet. World Health Organization. 2016. https://www.who.int/en/news-room/factsheets/detail/sexually-transmitted-infections-(stis). Accessed 20 Jun 2020

2. Silva LC, Miranda AE, Batalha RS, Sabino CC, Dib E, Talhari S. Chlamydia trachomatis infection among HIV-infected women attending an AIDS clinic in the city of Manaus, Brazil. Braz J Infect Dis. 2012:16:335-8.

3. Brunham RC, Rey-Ladino J. Immunology of chlamydia infection: implications for a chlamydia trachomatis vaccine. Nat Rev Immunol. 2005;2:149-61.

4. Paavonen J. Chlamydia trachomatis infections of the female genital tract: state of the art. Ann Med. 2012:44:18-28.

5. Abdelrahman YM, Belland RJ. The chlamydial developmental cycle. FEMS Microbiol Rev. 2005;29:949-59.

6. Bastidas RJ, Elwell CA, Engel JN, Valdivia RH. Chlamydial intracellular survival strategies. Cold Spring Harb Perspect Med. 2013;3:a010256.

7. Beatty WL, Belanger TA, Desai AA, Morrison RPBG. Tryptophan depletion as a mechanism of gamma interferon-mediated chlamydial persistence. Infect Immun. 1994:62:3705-511.

8. Schoborg RV. Chlamydia persistence - a tool to dissect chlamydia-host interactions. Microbes Infect. 2011;13:649-62.

9. Elwell C, Mirrashidi K, Engel J. Chlamydia cell biology and pathogenesis. Nat Rev Microbiol. 2016;14:385-400.

10. Ohmer M, Tzivelekidis T, Niedenfuhr N, Volceanov-Hahn L, Barth S, Vier J, et al. Infection of HeLa cells with chlamydia trachomatis inhibits protein synthesis and causes multiple changes to host cell pathways. Cell Microbiol. 2019:21:e12993.

11. Kading N, Szaszak M, Rupp J. Imaging of chlamydia and host cell metabolism. Future Microbiol. 2014;9:509-21.

12. Shima K, Kaeding N, Ogunsulire IM, Kaufhold I, Klinger M, Rupp J. Interferon$\gamma$ interferes with host cell metabolism during intracellular chlamydia trachomatis infection. Cytokine. 2018;112:95-101.

13. Gondek DC, Olive AJ, Stary G, Starnbach MN. CD4+ T cells are necessary and sufficient to confer protection against chlamydia trachomatis infection in the murine upper genital tract. J Immunol. 2012;189:2441-9. 
14. Gondek DC, Roan NR, Starnbach MN. T cell responses in the absence of IFNgamma exacerbate uterine infection with chlamydia trachomatis. J Immunol. 2009;183:1313-9.

15. Valdivia RH. Chlamydia effector proteins and new insights into chlamydial cellular microbiology. Curr Opin Microbiol. 2008;11:53-9.

16. Saka HA, Thompson JW, Chen YS, Kumar Y, Dubois LG, Moseley MA, Valdivia $\mathrm{RH}$. Quantitative proteomics reveals metabolic and pathogenic properties of chlamydia trachomatis developmental forms. Mol Microbiol. 2011;82:1185203.

17. Belland RJ, Nelson DE, Virok D, Crane DD, Hogan D, Sturdevant D, Beatty WL, Caldwell HD. Transcriptome analysis of chlamydial growth during IFNgamma-mediated persistence and reactivation. Proc Natl Acad Sci U S A. 2003;100:15971-6.

18. Albrecht M, Sharma CM, Reinhardt R, Vogel J, Rudel T. Deep sequencingbased discovery of the chlamydia trachomatis transcriptome. Nucleic Acids Res. 2010;38:868-77.

19. Abdelrahman YM, Rose LA, Belland RJ. Developmental expression of noncoding RNAs in chlamydia trachomatis during normal and persistent growth. Nucleic Acids Res. 2011;39:1843-54.

20. Porcella SF, Carlson JH, Sturdevant DE, Sturdevant GL, Kanakabandi K, Virtaneva K, et al. Transcriptional profiling of human epithelial cells infected with plasmid-bearing and plasmid-deficient Chlamydia trachomatis. Infect Immun. 2015;83:534-43.

21. Humphrys MS, Creasy T, Sun Y, Shetty AC, Chibucos MC, Drabek EF. Simultaneous transcriptional profiling of Bacteria and their host cells. PLoS One. 2013;8:e80597.

22. Carabeo RA, Dooley CA, Grieshaber SS, Hackstadt T. Rac interacts with Abi-1 and WAVE2 to promote an Arp2/3-dependent actin recruitment during chlamydial invasion. Cell Microbiol. 2007;9:2278-88.

23. George Z, Omosun Y, Azenabor AA, Partin J, Joseph K, Ellerson D, et al. The roles of unfolded protein response pathways in chlamydia pathogenesis. J Infect Dis. 2017:215:456-65.

24. Wu HZ, Ong KL, Seeher K, Armstrong NJ, Thalamuthu A, Brodaty $H$, et al. Circulating microRNAs as biomarkers of Alzheimer's disease: a systematic review. J Alzheimers Dis. 2016;49:755-66.

25. Alevizos I, Illei GG. MicroRNAs as biomarkers in rheumatic diseases. Nat Rev Rheumatol. 2010;6:391-8.

26. Bhomia M, Balakathiresan NS, Wang KK, Papa L, Maheshwari RK. A panel of serum MiRNA biomarkers for the diagnosis of severe to mild traumatic brain injury in humans. Sci Rep. 2016;6:28148.

27. Guo X, Gao L, Liao Q. Long non-coding RNAs function annotation: a global prediction method based on bi-colored networks. Nucleic Acids Res. 2013; 41:e35.

28. Chen L, Dzakah EE, Shan G. Targetable long non-coding RNAs in cancer treatments. Cancer Lett. 2018;418:119-24.

29. Dzakah EE, Waqas A, Wei S, Yu B, Wang X, Fu T, Shan G. Loss of miR-83 Extends Lifespan and Affects Target Gene Expression in an Age-Dependent Manner in Caenorhabditis Elegans. J Genet Genomics. 2018;45:651-62.

30. Xia M, Bumgarner RE, Lampe MF, Stamm WE. Chlamydia trachomatis infection alters host cell transcription in diverse cellular pathways. J Infect Dis. 2003;187:424-34

31. Di Pietro M, Filardo S, Romano S, Sessa R. Chlamydia trachomatis and chlamydia pneumoniae interaction with the host: latest advances and future prospective. Microorganisms. 2019;7:140.

32. Buchholz KR, Stephens RS. The extracellular signal-regulated kinase/ mitogen-activated protein kinase pathway induces the inflammatory factor interleukin-8 following chlamydia trachomatis infection. Infect Immun. 2007; 75:5924-9.

33. Gurumurthy RK, Maurer AP, Machuy N, Hess S, Pleissner KP, Schuchhardt J, et al. A loss-of-function screen reveals Ras- and Raf-independent MEK-ERK signaling during Chlamydia trachomatis infection. Sci Signal. 2010;3:ra21.

34. Scidmore MA. Cultivation and laboratory maintenance of chlamydia trachomatis. Curr Protoc Microbiol. 2005:00:11A.1.1-11A.1.25.

35. Hu S, Wang $X$, Shan G. Insertion of an Alu element in an IncRNA leads to primate specific modulation of alternative splicing. Nat Struct Biol. 2016;23: 1011-9.

36. Bioinformatics B. FastQC-A quality control tool for high throughput sequence data. 2016. Available from: FastQCC Babraham Bioinformatics 2016. http://www.bioinformatics.babraham.ac.uk/projects/fastqc/. Accessed 10 Oct 2019.
37. Anders S, Huber W. Differential expression analysis for sequence count data. Genome Biol. 2010;11:R106.

38. Eran E, Roy N, Israel S, Doron L, Zohar Y. Gorilla: a tool for discovery and visualization of enriched $\mathrm{GO}$ terms in ranked gene lists. BMC Bioinform. 2009; 10:48.

39. Kuleshov MV, Jones MR, Rouillard AD, Fernandez FN, Duan Q, Wang Z, et al. Enrichr: a comprehensive gene set enrichment analysis web server 2016 update. Nucleic Acids Res. 2016;44:W90-7.

40. Shannon P, Markiel A, Ozier O. Cytoscape: a software environment for integrated models of biomolecular interaction networks. Genome Res. 2003 13:2498-504.

\section{Publisher's Note}

Springer Nature remains neutral with regard to jurisdictional claims in published maps and institutional affiliations.
Ready to submit your research? Choose BMC and benefit from:

- fast, convenient online submission

- thorough peer review by experienced researchers in your field

- rapid publication on acceptance

- support for research data, including large and complex data types

- gold Open Access which fosters wider collaboration and increased citations

- maximum visibility for your research: over $100 \mathrm{M}$ website views per year

At $\mathrm{BMC}$, research is always in progress.

Learn more biomedcentral.com/submissions 